\title{
MYOCARDIAL FIBROSIS WITH SEVERE IMPAIRMENT OF CARDIAC FUNCTION IN LIMITED CUTANEOUS SYSTEMIC SCLEROSIS: CASE REPORT
}

Igor Beltrão Duarte Fernandes ${ }^{1, \star}$, Luiza Sá e Rêgo Tupinambá ${ }^{1}$, Germana Ribeiro de Araújo Carneiro de Lucena ${ }^{1}$, Mariana Davim Ferreira Gomes $^{1}$, Edgard Torres dos Reis Neto ${ }^{1}$

1.Universidade Federal de São Paulo, São Paulo (SP), Brazil.

*Corresponding author: igorbdfernandes@outlook.com

\section{BACKGROUND}

Cardiac manifestations in systemic sclerosis (SSc) are variable and increased morbidity and mortality in the disease. It is more frequently in the diffuse cutaneous form of SSc and includes macrovascular coronary artery disease, conduction defects and tachyarrhythmias, autonomic insufficiency, pericardial and myocardial involvement and heart failure. Myocardial fibrosis is a rare manifestation, even more in the limited cutaneous SSc form of the disease.

\section{CASE REPORT}

A 29-year-old male patient reported progressively dyspnea for 2 months with worsening in the last 2 weeks, orthopnea, paroxysmal nocturnal dyspnea, weight gain and edema of lower limbs. Physical examination revealed jugular venous distension, signs of pulmonary congestion and inadequate peripheral perfusion with diagnosis of heart failure. As personal antecedent, he was diagnosed at 12 years old with limited cutaneous SSc (without visceral involvement) and did not present any cardiovascular risk factors (smoke, hypertension, dyslipidemia, family history and coronary artery disease) or use of illicit drugs. Laboratory tests revealed cardiac troponin $460 \mathrm{ng} / \mathrm{mL}$ (normal until $14 \mathrm{ng} / \mathrm{mL}$ ), ANA 1:1280 homogeneous pattern with negative anti-ENA and anti-dsDNA antibodies and normal complement levels. Electrocardiogram did not demonstrate signs of ischemia or pericarditis. Echocardiography showed increased dimensions in right and left atria, moderate dilation of the left ventricle, significant degree of impairment of systolic function, with akinesia at septum, inferior wall and hypokinesis of the other walls and ejection fraction of left ventricle of $22 \%$. Reduced right ventricular function. Cardiac magnetic resonance (CMR) showed fibrosis in the subendocardial region ( $<50 \%$ ), with ischemic pattern in basal segment of inferior and inferolateral leads of the left ventricle and focal area of fibrosis at insertion of septum ventricular. Coronary computed tomography angiography was normal.

\section{CONCLUSION}

Myocardial fibrosis has been described in 50-80\% of SSc with cardiac involvement with most of the patients with subclinical disease. Recently, with the advent of noninvasive imaging techniques, such as CMR, early diagnosis has becoming more effective. Heart disease is associated with a greater mortality risk and, although the proportion of deaths in SSc due to lung or kidney damage has gradually declined, cardiac-related deaths remain stable, accounting for approximately $15 \%$ of all deaths. 